\title{
Transport coefficients of the QGP
}

\author{
Alessandro Amato* \\ Department of Physics, College of Science, Swansea University, Swansea, United Kingdom \\ Institut für theoretische Physik, Universität Regensburg, Regensburg, Germany \\ E-mail: Alessandro. Amato@physik.uni-regensburg.de
}

\section{Gert Aarts}

Department of Physics, College of Science, Swansea University, Swansea, United Kingdom E-mail: g.aarts@swan.ac.uk

\section{Chris Allton}

Department of Physics, College of Science, Swansea University, Swansea, United Kingdom E-mail: c.alltoneswan.ac.uk

\section{Pietro Giudice}

Universität Münster, Institut für Theoretische Physik, Münster, Germany

E-mail: p.giudiceduni-muenster.de

\section{Simon Hands}

Department of Physics, College of Science, Swansea University, Swansea, United Kingdom E-mail: s.handseswansea.ac.uk

\section{Jon-Ivar Skullerud}

Department of Mathematical Physics, National University of Ireland Maynooth, Maynooth, County Kildare, Ireland

E-mail: jonivarethphys.nuim.ie

The FASTSUM collaboration presents a study on the temperature dependence of the electrical conductivity $\sigma$ in the quark-gluon plasma, using the methods of lattice QCD. Correlators of the exactly conserved vector current are measured at different temperatures across the deconfinement transition, using ensembles of $2+1$ flavours of dynamical fermions on anisotropic lattices. We then employ bayesian methods (MEM) to extract the relevant spectral functions, which are found to be consistent with $\sigma / T$ rising as a function of $T$. The robustness of the results is verified by a detailed analysis of the systematics involved in the bayesian reconstruction of the spectral functions.

31st International Symposium on Lattice Field Theory - LATTICE 2013

July 29 - August 3, 2013

Mainz, Germany

\footnotetext{
*Speaker.
} 


\section{Introduction}

In this work we study the dynamical properties of the quark-gluon plasma (QGP), and in particular we focus on the transport phenomena associated with electric charge [1]. From an experimental point of view, a reliable first-principles determination of the conductivity is necessary to estimate the evolution of electromagnetic fields during heavy-ion collision experiments, where particle emission from the fireball is heavily influenced by the presence of a magnetic field [2]. In order to understand the real-time evolution of the plasma, effective models, such as dissipative hydrodynamics, are used [3]. Transport coefficients, e.g. the bulk/shear viscosity and the electrical conductivity, enter as parameters in these models and can be seen as low-energy variables describing the effects of the underlying quantum field theory. There are many phenomenological indications that the plasma produced in such collisions is strongly interacting. In many cases this leads to a very poor convergence of perturbation theory when used as a tool to derive expressions for transport coefficients directly from QCD, see e.g. results for the shear viscosity [4]. This is why we take here the lattice QCD approach, where these quantities may be extracted from nonperturbatively computed euclidean correlators. For recent reviews, see e.g. Ref. [5].

\section{Electrical Conductivity on the Lattice}

In the following we will describe how to formulate the problem of calculating the electrical conductivity on the lattice. As a starting point, we consider the contribution from the up and down quarks to the electromagnetic current:

$$
j_{\mu}^{\mathrm{em}}(x)=\frac{2}{3} e j_{\mu}^{\mathrm{up}}(x)-\frac{1}{3} e j_{\mu}^{\mathrm{down}}(x)
$$

where $j_{\mu}^{f}$ is the vector current for each flavour considered, the coefficients in front represent the fractional charge of the quark and $e$ is the elementary charge. The connection between the conductivity and lattice QCD is provided by the spectral function $\rho_{\mu v}(\omega)$, which is defined through the euclidean correlator of the electromagnetic current (2.1) as follows:

$$
G_{\mu \nu}^{\mathrm{em}}(\tau)=\int d^{3} x\left\langle j_{\mu}^{\mathrm{em}}(\tau, \mathbf{x}) j_{\nu}^{\mathrm{em}}(0, \mathbf{0})^{\dagger}\right\rangle=\int_{0}^{\infty} \frac{d \omega}{2 \pi} \rho_{\mu \nu}^{\mathrm{em}}(\omega) \frac{\cosh [\omega(\tau-1 / 2 T)]}{\sinh [\omega / 2 T]},
$$

where $\tau$ is the euclidean time coordinate, $\omega$ the frequency and we performed the projection to zero momentum. On the r.h.s. the integral transform has a nontrivial kernel $K(\tau, \omega)$, which depends on the temperature $T$. The operation of obtaining the spectral function $\rho_{\mu v}(\omega)$ from the euclidean correlator $G_{\mu \nu}^{\mathrm{em}}(\tau)$, i.e. inverting Eq. (2.2), represents the main computational challenge as it involves analytical continuation from euclidean to Minkowski space. Our approach is described in Sec. 4. Once the spectral function is available, the electrical conductivity $\sigma$ can be obtained by taking the low-frequency limit, known as Kubo's formula [6]:

$$
\frac{\sigma}{T}=\frac{1}{6 T} \lim _{\omega \rightarrow 0} \frac{\rho^{\mathrm{em}}(\omega)}{\omega}, \quad \rho^{\mathrm{em}}(\omega)=\sum_{i=1}^{3} \rho_{i i}^{\mathrm{em}}(\omega)
$$




\begin{tabular}{cccccc}
\hline \hline$N_{s}$ & $N_{\tau}$ & $T[\mathrm{MeV}]$ & $T / T_{c}$ & $N_{\mathrm{CFG}}$ & $N_{\mathrm{SRC}}$ \\
\hline \hline 32 & 16 & 352 & 1.90 & 1059 & 4 \\
24 & 20 & 281 & 1.52 & 1001 & 4 \\
32 & 24 & 235 & 1.27 & 500 & 4 \\
32 & 28 & 201 & 1.09 & 502 & 4 \\
32 & 32 & 176 & 0.95 & 501 & 4 \\
24 & 36 & 156 & 0.84 & 501 & 4 \\
24 & 40 & 141 & 0.76 & 523 & 4 \\
32 & 48 & 117 & 0.63 & 601 & 1 \\
\hline \hline
\end{tabular}

(a) Gauge configurations.

\begin{tabular}{rl}
\hline \hline$a_{s}[\mathrm{fm}]$ & $0.1227(8)$ \\
$a_{\tau}[\mathrm{fm}]$ & $0.03506(23)$ \\
$\xi=a_{s} / a_{\tau}$ & 3.5 \\
$\gamma_{g}$ & 4.3 \\
$\gamma_{f}$ & 3.4 \\
$c_{t}$ & 0.9027 \\
$c_{s}$ & 1.5893 \\
$\hat{m}_{\mathrm{u}, \mathrm{d}}$ & -0.0840 \\
$\hat{m}_{\mathrm{s}}$ & -0.0743 \\
\hline \hline
\end{tabular}

(b) Simulation parameters.

Table 1: (a) The gauge ensembles have lattice size of $N_{s}^{3} \times N_{\tau}$, with $N_{\mathrm{CFG}}$ configurations available for each set and a number of $N_{\mathrm{SRC}}$ sources for the analysis. (b) The parameters in the action (3.1).

\section{Simulation Details}

In contrast to lattice simulations used to study QCD thermodynamics, where the staggered formulation is often preferred, we take here the choice of clover-improved Wilson fermions, with $2+1$ flavors [7]. This is motivated by the fact that matching the physical degrees of freedom is much easier. In fact, with staggered fermions, the euclidean correlator described above, which represents our main probe of the QGP, contains a signal from an opposite parity partner, effectively reducing the number of usable points in the temporal direction [8]. This is undesired, since a higher resolution in $G_{\mu \nu}^{\mathrm{em}}(\tau)$ brings a more reliable analysis. These requirements, together with the need to keep the computational cost under control, have motivated us to simulate using anisotropic lattices, where the lattice spacing $a_{\tau}$ in the time direction is taken to be smaller than the spatial one $a_{s}$. The drawback of this choice is the appearance of new bare parameters in the action, which have to be tuned carefully. This has been achieved in Ref. [7], to which we refer for further details. The gauge action is Symanzik and tadpole improved with tree-level coefficients. The Dirac operator reads:

$$
D[U]=\hat{m}_{0}+\gamma_{4} \hat{W}_{4}+\frac{1}{\gamma_{f}} \sum_{i} \gamma_{i} \hat{W}_{i}-\frac{c_{t}}{2} \sum_{i} \sigma_{4 i} \hat{F}_{4 i}-\frac{c_{s}}{2 \gamma_{g}} \sum_{i<j} \sigma_{i j} \hat{F}_{i j}
$$

where $\hat{m}_{0}$ and $\hat{W}_{\mu}$ are the mass and the usual Wilson operator and $\gamma_{\mu}$ are the Dirac matrices. The last two terms are the clover operators, with $\sigma_{\mu \nu}=\frac{i}{2}\left[\gamma_{\mu}, \gamma_{\nu}\right]$ and $\hat{F}_{\mu \nu}$ the lattice version of the field strength tensor. Their coefficients $c_{t, s}$ have been chosen according to tree-level conditions. The novel parameters mentioned above are the bare gauge $\left(\gamma_{g}\right)$ and fermion $\left(\gamma_{f}\right)$ anisotropies. These are tuned in Ref. [7], giving a ratio $\xi \equiv a_{s} / a_{\tau}=3.5$. The gauge links $U_{\mu}$ are represented by threedimensional stout-smeared links [9], with smearing weight $\rho=0.14$ and $n_{\rho}=2$ iterations. The light and strange quark mass parameters are chosen [7] to reproduce the physical strange quark mass and a light quark mass with $M_{\pi} / M_{\rho}=0.446(3)$. The numerical value of all the parameters appearing in Eq. (3.1) can be found in Table 1b.

We have generated a number of finite-temperature ensembles, using a fixed lattice spacing approach. This allows for a determination of the temperature dependence of the conductivity, 

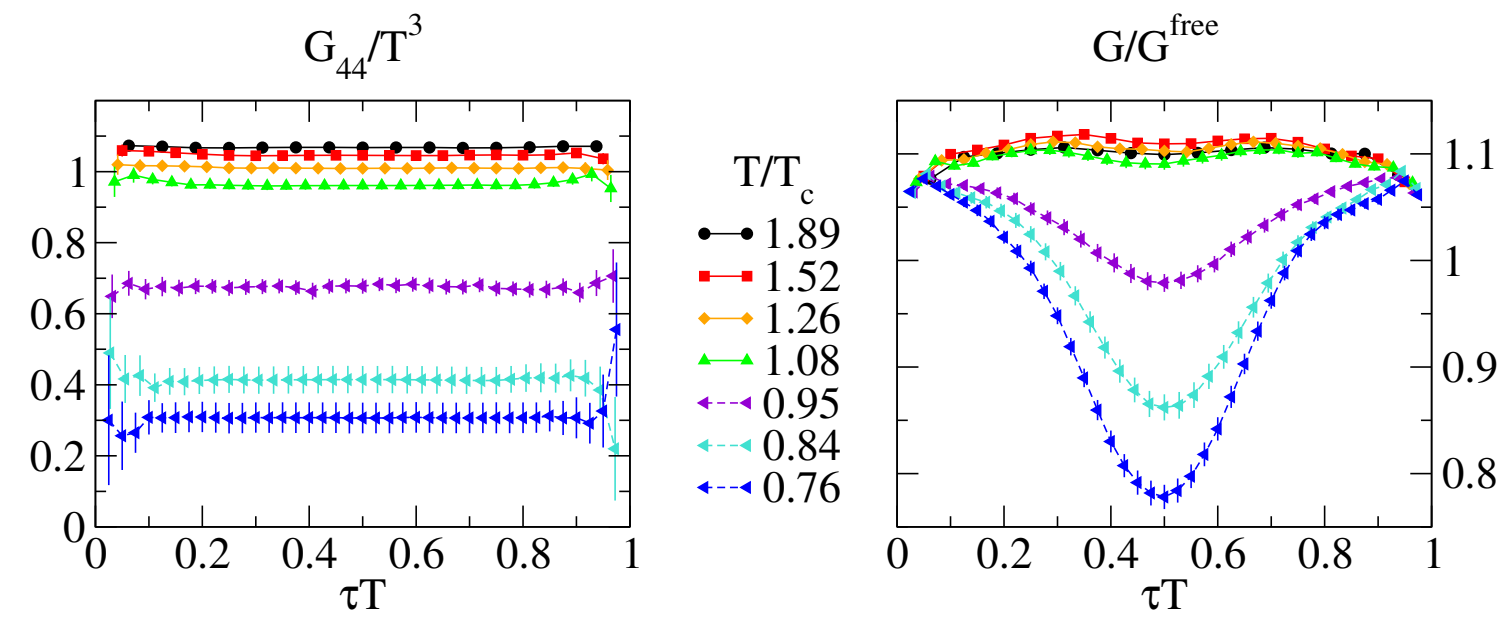

Figure 1: Diagonal components of the correlator (2.2). Dashed (solid) lines represent temperatures below (above) $T_{c}$. Left: temporal component $G_{44}$ rescaled by $T^{3}$. Right: spatial component $G(\tau)=$ $\sum_{i} G_{i i}(\tau)$, normalized by its value in the free case.

missing in previous studies. The critical temperature is estimated from the renormalized Polyakov loop inflection point [10], see Table 1a. We use the exactly conserved vector current on the lattice:

$$
V_{\mu}^{\mathrm{C}}(x)=\kappa_{\mu}\left[\bar{\psi}(x+\hat{\mu})\left(1+\gamma_{\mu}\right) U_{\mu}^{\dagger}(x) \psi(x)-\bar{\psi}(x)\left(1-\gamma_{\mu}\right) U_{\mu}(x) \psi(x+\hat{\mu})\right]
$$

where $\kappa_{4}=1 / 2, \kappa_{i}=1 /\left(2 \gamma_{f}\right)$. To compute the correlator (2.2), we use Wick contractions and neglect disconnected diagrams. This is justified by the fact that their contribution is identically zero in the $N_{f}=3$ case, and we note that the same choice has been applied in all previous studies $[8,11,12]$. Since the up and down quark fields in Eq. (2.1) are degenerate, we can factor out their fractional charge assignments, via $C_{\mathrm{em}}=e^{2}\left(q_{u}^{2}+q_{d}^{2}\right)=5 / 9 e^{2}$ and define $G_{\mu \nu}^{\mathrm{em}}(\tau)=C_{\mathrm{em}} G_{\mu \nu}(\tau)$. Similarly in Eq. (2.3) we define $\rho^{\mathrm{em}}(\omega)=C_{\mathrm{em}} \rho(\omega)$. We show in Fig. 1 the diagonal components of $G_{\mu v}(\tau)$. Above $T_{c}$, correlators measured in ensembles of increasing temperature show little differences between each other, while below $T_{c}$ their behaviour rapidly changes with $T$.

\section{Maximum Entropy Method}

In this section we describe the method used to obtain the spectral function. At large $\omega$, the kernel $K(\tau, \omega)$ in Eq. (2.2) is highly suppressed, which allows us to cut off the integral at some $\omega_{\max }$. We then discretise the interval $0<\omega<\omega_{\max }$ using a stepsize $\Delta \omega$ with $N_{\omega}=\omega_{\max } / \Delta \omega$. The resulting equation is $G\left(\tau_{i}\right)=\Delta \omega \sum_{j=0}^{N_{\omega}} K_{i j} \rho_{j}$, where the correlator $G\left(\tau_{i}\right)$ is defined over a number of euclidean points of around $N_{\tau} / 2 \sim O(10-20)$. On the other hand, the discretised spectral function $\rho_{j}$ is defined over $N_{\omega} \sim O\left(10^{3}\right)$ points. This is often referred to as an ill-posed problem.

A search for the solution using standard $\chi^{2}$ methods would fail, because the result would not be unique. One way to proceed is to use a physically motivated Ansatz for the spectral function, with a number of parameters to be minimized. This path has been followed in Ref. [12]. Here we use a bayesian analysis, the Maximum Entropy Method (MEM), which was first applied to lattice 

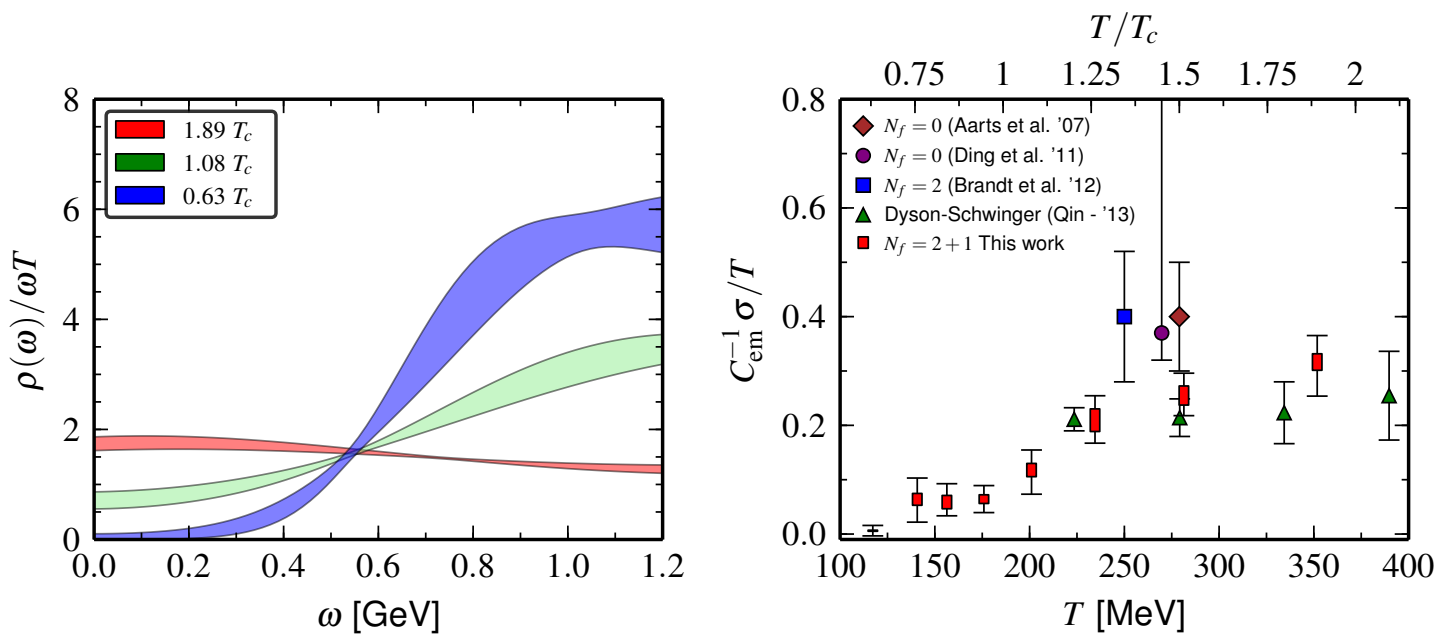

Figure 2: Left: spectral functions $\rho(\omega) / \omega T$ at different $T$ 's. The intercept is proportional to $\sigma / T$, see Eq. (2.3). Right: temperature dependence of $C_{\mathrm{em}}^{-1} \sigma / T$. The vertical size of the rectangles reflects the systematic uncertainty due to changes in the default model, while the error bars include the statistical jackknife error as well. Previous results $[8,12]$ are indicated: the $N_{f}=0$ points are inserted matching the values of $T / T_{c}$. The triangles represent a Dyson-Schwinger result [14].

QCD in Ref. [13]. This consists of extracting the most probable spectral function $\rho(\omega)$, given some prior knowledge $H$ and the data $D$. This is expressed as a conditional probability:

$$
P[\rho \mid D H]=\frac{P[D \mid \rho H] P[\rho \mid H]}{P[D \mid H]} \propto \exp (-L+\alpha S),
$$

where $L$ is the standard likelihood function and $S$ is the Shannon-Jaynes entropy, given by:

$$
S=\int_{0}^{\infty} \frac{d \omega}{2 \pi}\left[\rho(\omega)-m(\omega)-\rho(\omega) \ln \frac{\rho(\omega)}{m(\omega)}\right]
$$

where $m(\omega)=m_{0}(b+\omega) \omega$ is the default model, representing our prior information on $\rho(\omega)$. Here $m_{0}$ is an overall normalization and the parameter $b$ is algorithmically crucial to allow a non-zero conductivity $\sigma$, which is obtained from the intercept $\lim _{\omega \rightarrow 0} \rho(\omega) / \omega$, as shown in Eq. (2.3). The result is then obtained by maximising $P[\rho \mid D H]$, where we use a modification of Bryan's algorithm [8] which fixes kernel instabilities at low $\omega$.

\section{Results}

In Fig. 2 (left) we show the spectral function, $\rho(\omega) / \omega T$ for three different temperatures. The intercept is proportional to $\sigma / T$, which is shown in Fig. 2 (right) for temperatures across the deconfining transition. We also compare our $N_{f}=2$ result to previous findings on the lattice $[8,12]$ and to results obtained using Dyson-Schwinger equations [14].

We have carried out a series of tests in order to check the reliability of the MEM reconstruction of $\rho(\omega)$. In primis, the choice of default model, aka the specific value of $b$ should not be reflected in the final result for $\sigma$. In Fig. 3 (right) we explicitly check this by varying $b$ in the MEM analysis, observing stability provided that $b \gtrsim 0.4$. 

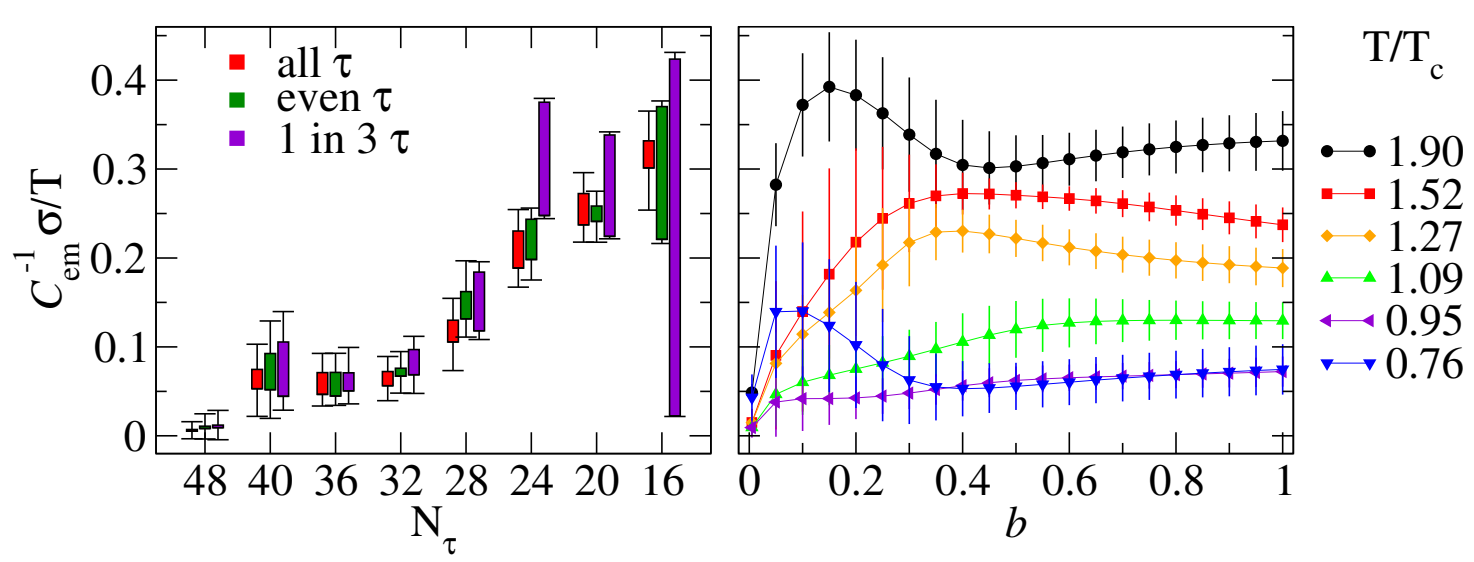

Figure 3: Stability tests. Left: $C_{\mathrm{em}}^{-1} \sigma / T$ when only a subset of the available time slices is used. Right: dependence on the parameter $b$ in the default model. Stability is achieved for $b \gtrsim 0.4$.
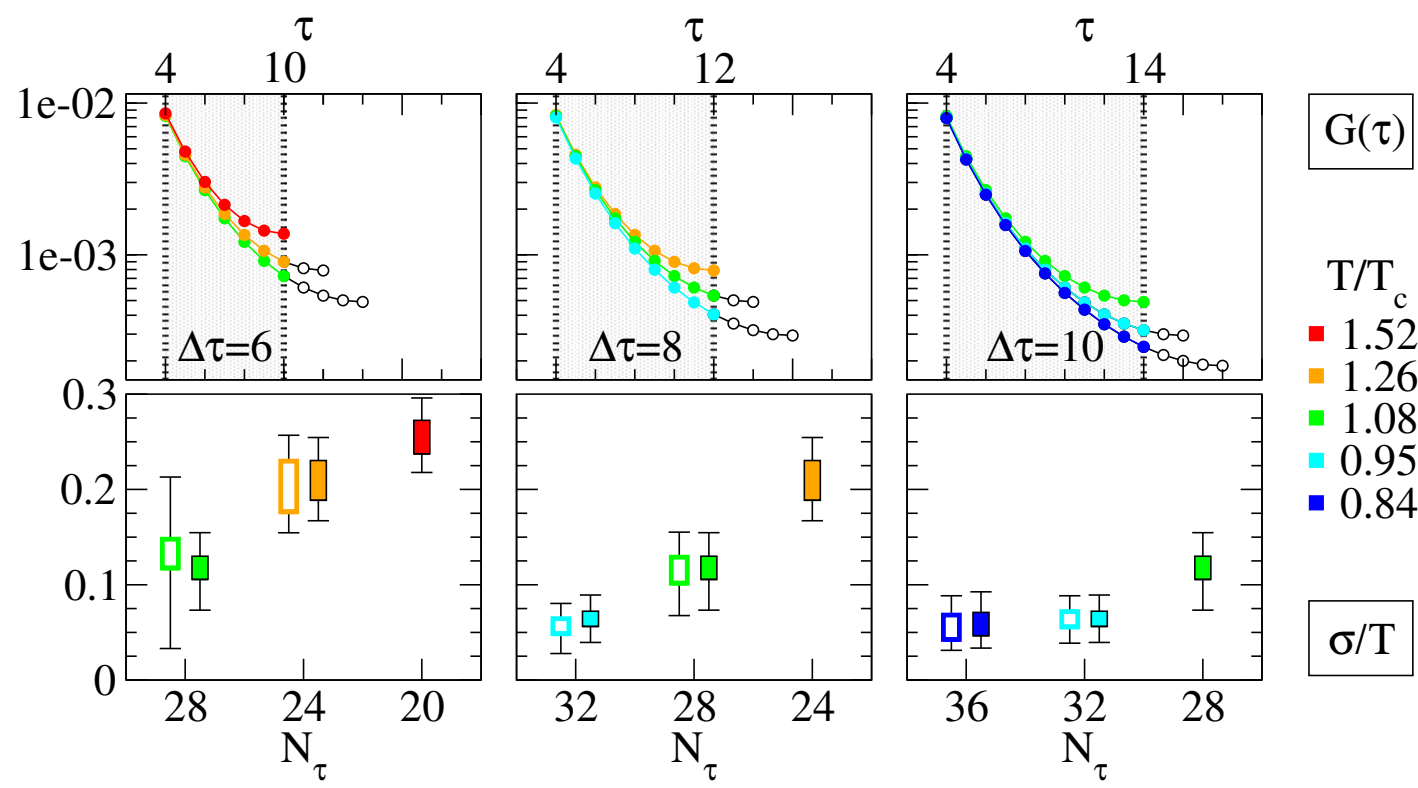

$\mathrm{T} / \mathrm{T}_{\mathrm{c}}$

$-1.52$

$-1.26$

$-1.08$

$-0.95$

- 0.84

Figure 4: Stability tests discarding the last time slices in the correlator. Top: correlators with used time slices indicated. Bottom: corresponding MEM results for $C_{\mathrm{em}}^{-1} \sigma / T$. Open symbols use the restricted time range $\Delta \tau$ shown in the upper pane, full symbols use the entire time range available.

In Sec. 3 we mentioned the importance of having a high resolution in the correlator $G(\tau)$ and how this was achieved by the introduction of the anisotropy. To justify this choice, we run MEM using only a subset of the available time slices in the correlator, and check whether the result is stable. This is shown in Fig. 3 (left), where it is clear that for high temperatures, the anisotropy is crucial to extract a signal for the conductivity. For colder ensembles the result is instead stable.

As also pointed out in Sec. 3, the number of euclidean points available in the correlator will decrease as the temperature is raised. One might think that this difference in the extent of $G(\tau)$ is responsible for the $T$ dependence of the conductivity, rather than it being a genuine thermal effect. To show that this is not the case, we run the MEM analysis on correlators at different temperatures, 
but constrained to have the same number of time slices, which is achieved by systematically discarding the last points of colder ensembles. A graphical representation of the procedure is showed in Fig. 4. We observe excellent stability as the euclidean time range is varied.

\section{Conclusions}

We have presented the first lattice QCD calculation of the temperature dependence of the electrical conductivity $\sigma$ divided by $T$, using the conserved current and anisotropic lattices [1]. We found that $\sigma / T$ increases with temperature. In the near future we plan to include the contribution from the strange quark and also evaluate the charge diffusion constant $D$, combining the results for $\sigma$ with those for the electric charge susceptibility [15].

\section{Acknowledgments}

This work was supported by STFC, UKQCD and the STFC funded DiRAC Facility, the Royal Society, the Wolfson Foundation, the Leverhulme Trust and the European Union Grant Agreement number 238353 (ITN STRONGnet).

\section{References}

[1] A. Amato, G. Aarts, C. Allton, P. Giudice, S. Hands and J. -I. Skullerud, Phys. Rev. Lett. 111 (2013) 172001, [hep-lat/1307.6763].

[2] K. Tuchin, arXiv:1301.0099 [hep-ph]; L. McLerran and V. Skokov, arXiv:1305.0774 [hep-ph].

[3] U. W. Heinz and R. Snellings, arXiv:1301.2826 [nucl-th]; C. Gale, S. Jeon and B. Schenke, Int. J. Mod. Phys. A 28, 1340011 (2013).

[4] P. B. Arnold, G. D. Moore and L. G. Yaffe, JHEP 0011, 001 (2000) [hep-ph/0010177].

[5] G. Aarts, PoS LAT 2007 (2007) 001; T. Schäfer and D. Teaney, Rept. Prog. Phys. 72 (2009) 126001; H. B. Meyer, Eur. Phys. J. A 47 (2011) 86.

[6] L. P Kadanoff and P. C. Martin, Annals of Physics, 24 (1963) 419-469.

[7] H. -W. Lin et al. [Hadron Spectrum Collaboration], Phys. Rev. D 79 (2009) 034502; R. G. Edwards, B. Joo and H. -W. Lin, Phys. Rev. D 78 (2008) 054501.

[8] G. Aarts, C. Allton, J. Foley, S. Hands and S. Kim, Phys. Rev. Lett. 99 (2007) 022002.

[9] C. Morningstar and M. J. Peardon, Phys. Rev. D 69 (2004) 054501.

[10] J.-I. Skullerud et al, in preparation.

[11] S. Gupta, Phys. Lett. B 597, 57 (2004).

[12] H. -T. Ding, A. Francis, O. Kaczmarek, F. Karsch, E. Laermann et al, Phys. Rev. D 83 (2011) 034504; B. Brandt, A. Francis, H. B. Meyer and H. Wittig, JHEP 1303 (2013) 100.

[13] M. Asakawa, T. Hatsuda and Y. Nakahara, Prog. Part. Nucl. Phys. 46, 459 (2001).

[14] S. Qin, arXiv:1307.4587 [nucl-th].

[15] P. Giudice, G. Aarts, C. Allton, A. Amato, S. Hands and J. -I. Skullerud, arXiv:1309.6253 [hep-lat]. 